\title{
Peptide Dendrimer-Lipid Conjugates as DNA and siRNA Transfection Reagents: Role of Charge Distribution Across Generations
}

\author{
Marc Heitz $\S^{\star}$, Albert Kwok, Gabriela A. Eggimann, Florian Hollfelder, Tamis Darbre, \\ and Jean-Louis Reymond*
}

§SCS-DSM Award for best poster presentation in Medicinal Chemistry \& Chemical Biology

\begin{abstract}
Transfection reagents are used to deliver DNA and siRNA into cells to achieve genetic manipulations, and may ultimately enable nonviral gene therapy. Progress in transfection reagents is limited by the fact that such reagents cannot be easily optimized due to their polymeric nature and/or difficult synthesis. We have developed a new class of well-defined and easily modifiable transfection reagents in the form of peptide dendrimers. These dendrimers self-assemble with DNA or siRNA and lipofectin to form nanoparticles which efficiently enter mammalian cells and liberate their nucleic acid cargo. By systematically modifying the amino acid sequence of our dendrimers we have found that their transfection efficiency depends on the distribution of positive charges and hydrophobic residues across the dendrimer branches. Positive charges present in all three generations lead to efficient DNA delivery, whereas siRNA delivery requires charges in the outer two generations combined with a hydrophobic dendrimer core.
\end{abstract}

Keywords: Dendrimer $\cdot$ DNA $\cdot$ Peptide $\cdot$ siRNA $\cdot$ Transfection

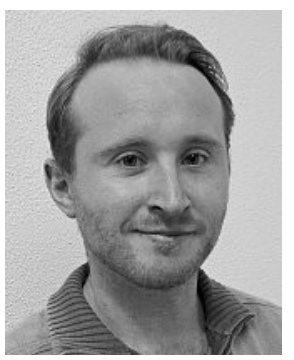

Marc Heitz received a BSc in chemistry (2010) and a combined MSc in organic chemistry at the University of Haute-Alsace and in medicinal chemistry and pharmacology at the University Joseph-Fourier (2012). His thesis included research in medicinal chemistry at Evolva SA (2011) and in structural biology in Prof. Dominique Housset laboratory (2012). He worked as a research assistant on solid phase synthesis in Prof. Kristian Strømgaard laboratory at University of Copenhagen (2012-2013). In 2014, he started his PhD on peptide dendrimer in Prof. Jean-Louis Reymond laboratory at University of Bern.

${ }^{*}$ Correspondence: M. Heitz, Prof. Dr. J-L. Reymond Department of Chemistry and Biochemistry

University of Bern

Freiestrasse 3, $\mathrm{CH}-3012$ Bern

E-mail: marc.heitz@dcb.unibe.ch,

jean-louis.reymond@dcb.unibe.ch

\section{Introduction}

The central dogma of molecular biology is the process of DNA transcribed to RNA that is translated to a functional protein, this being the basis of all known life. ${ }^{[1]}$ In order to perform genetic manipulations, some of these steps could be modified. Delivering DNA into the cells will allow them to transcribe it to RNA and translate it into a protein, called overexpression. In a different manner, RNA interference (RNAi) allows effective and specific silencing of a protein by a synthetic double stranded small interfering RNA (siRNA) achieving sequence-specific gene knockdown by promoting the degradation of complementary mRNA via RNA induced silencing complex (RISC). ${ }^{[2,3]}$

Transfection, the process of delivering nucleic acids (such as DNA, mRNA, siRNA or miRNA) into cells, is key to performing the above-mentioned genetic manipulations. Potential therapeutic applications are crucially dependent on DNA and siRNA internalization into the cytosol - a bottleneck of the process. Naked or chemically modified DNA or siRNA delivery is of limited application and therefore nanoparticles encapsulating molecules have been investigated as a more general method to bring nucleic acids into cells. Many possibilities have been considered with nucleic acids delivery and transfection based on lipids, ${ }^{[4-6]}$ polymers, ${ }^{[7,8]}$ nanoparticles, ${ }^{[9]}$ dendrimers, ${ }^{[10-12]}$ peptides, ${ }^{[13]}$ and peptide-lipid conjugates. ${ }^{[14-16]}$ In many cases, efficiency, toxicity and stability in serum need to be further improved. However, the molecular basis of the observed effects is hard to delineate. Structure-activity relationships that would provide the means to rationally manipulate the observed effects are rare.

Dendrimers are multibranched synthetic macromolecules with a variety of applications in technology and biomedicine. ${ }^{[17]}$ For several years our group has shown that well-defined dendrimers consisting of amino acid building blocks can be readily obtained by solid-phase peptide synthesis in pure form. These so-called peptide dendrimers display a variety of interesting functions such as enzyme-like catalysis, ${ }^{[18]}$ metal and cofactor binding, ${ }^{[19]}$ cell-penetration and drug delivery, ${ }^{[20]}$ and antimicrobial effects by acting on both biofilms and biological membranes. ${ }^{[21,22]}$ Due to their favorable properties as poly-cationic molecules, we investigated the ability of our dendrimers to complex nucleic acids and discovered that indeed peptide dendrimers can act as transfection reagents for DNA. These experiments pointed to G1,2,3-KL (6) as the best dendrimer for DNA transfection (Fig. 1). ${ }^{[23]}$ Further studies showed that 
the same dendrimers could also be used for siRNA transfection, with dendrimer G2,3KL (5) performing best in this case. ${ }^{[24]}$ Here we compare the DNA and siRNA transfection properties of the different dendrimers, and report an extension of siRNA transfection studies with additional dendrimers 15-20 featuring variations in the placement and nature of cationic residues in the dendrimer branches (Table 1).

\section{Results and Discussion}

\section{Peptide Dendrimers}

Our peptide dendrimer transfection reagents consist of three successive generations of dipeptides connected by branching lysine residues and forming a hydrophobic polycation featuring leucine or alanine as hydrophobic residues, lysines, arginines or histidines as cationic residues, and a conserved glycine-serine-cysteine tripeptide core. The sequences differ in the distribution of the different residues along the dendrimer generations. In addition to dendrimers 1-14 reported in our previous publication we have extended the series with the new sequences 15-20 (G1,3KL, Ac-G2,3-KL, G1,3-RL, H-G2,3-KL, G2,3-HL and G1,2,3-HL). All dendrimers are prepared by SPPS using Fmoc/Boc/t$\mathrm{Bu}$ protection, followed by acid cleavage from the resin and obtained in pure form after preparative HPLC. ${ }^{[25]}$

\section{DNA and siRNA Transfection}

Both the DNA and the siRNA transfections are performed using a very similar protocol. The complexes are formed by mixing the nucleic acid (DNA or siRNA) with a peptide dendrimer and lipofectin, which is a cationic/neutral helper lipid mixture consisting of 1,2-dioleyloxy- 3-trimethylammonium propane chloride (DOTMA) and 1,2-dioleoyl-glycero3-phosphatidylethanolamine (DOPE). ${ }^{[26]}$ The resulting dendriplexes are then used to treat mammalian HeLa cells in OptiMEM, a serum-free medium, for $4 \mathrm{~h}$ followed by 24 or $48 \mathrm{~h}$ incubation in full DMEM medium. To test DNA transfection efficiency we use the plasmid pCI-Luc, which codes for luciferase. ${ }^{[27,28]}$ This enzyme converts luciferin into oxyluciferin producing energy in the form of light. The efficiency of intracellular plasmid delivery by the different transfection reagents is measured as the level of luminescence produced by the enzyme, which is quantified by a luciferase assay kit and normalized to the levels observed with lipofectin only.

For siRNA transfection studies, our assay system targets GAPDH (glyceraldehyde 3-phosphate dehydrogenase), an enzyme converting glyceraldehyde 3-phosphate to 1,3-bisphosphoglyceric acid by the reduction of nicotinamide adenine dinucleotide $\left(\mathrm{NAD}^{+}\right)$to NADH and expressed in a majority of cell lines.[29] SiRNA (siGAPDH) knockdown is assessed with a KD Alert GAPDH assay kit, quantifying the amount of $\mathrm{NAD}^{+}$converted to NADH by measuring product fluorescence. This fluorescence value is then normalized to non-treated cells and compared to siNC, a negative control siRNA with a random sequence which does not target the GAPDH mRNA.

For both DNA and siRNA transfections the presence of lipofectin is absolutely required for efficient transfection by the peptide dendrimer. Indeed for DNA and siRNA delivery removing lipofectin leads to no luciferase expression ( 0 to $20 \%$ ) or GAPDH knockdown (77 to $117 \%$ ). An overview of DNA and siRNA transfection efficiencies by the different dendrimers studied is

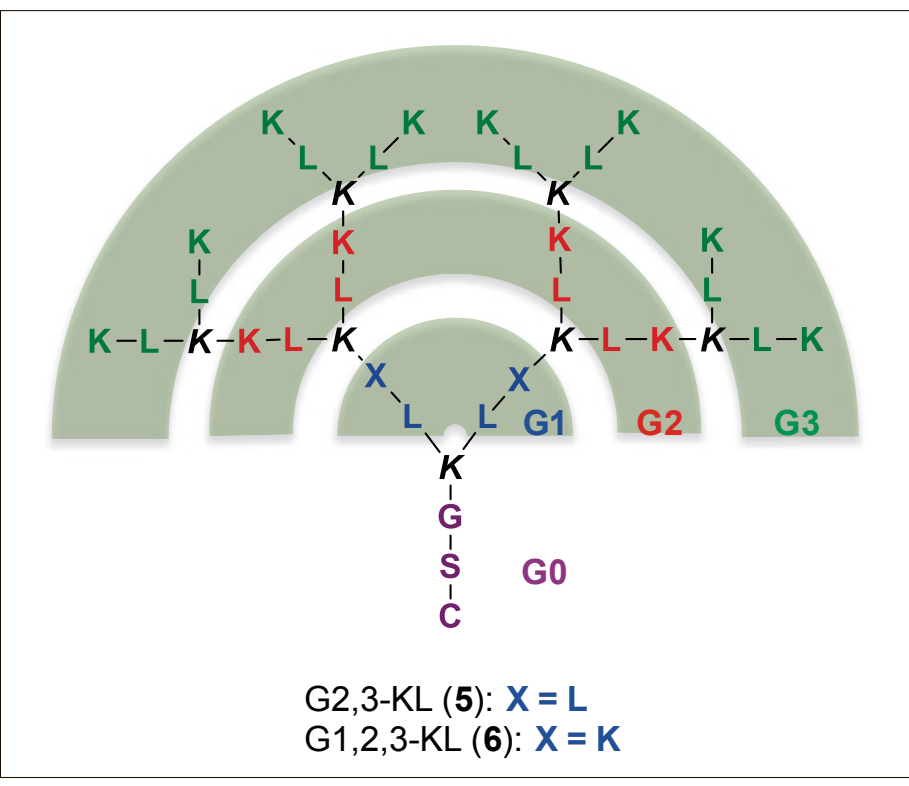

Fig. 1. Structures of peptide dendrimers G2,3-KL (5), which is an efficient siRNA transfection reagent, and $\mathrm{G} 1,2,3-\mathrm{KL}(\mathbf{6})$, which is an efficient DNA transfection reagent. Both dendrimers are used together with lipofectin. shown in Fig. 2. The best DNA transfection is observed with dendrimers G1,2,3$\mathrm{KL}$ (6) and G1,2,3-RL (9), indicating that positive charges are required across the three dendrimer generations. By contrast siRNA knockdown works best with G2,3KL (5) and G2,3-RL (8), which both contain a more hydrophobic G1 with a leucine dyad (LL). Increasing dendrimer size by means of disulfide bridge formation (1214) diminishes transfection efficiency for both DNA and siRNA. Dendrimers such as $\mathbf{1 0}$ containing alanine instead of leucine as hydrophobic residues do not work efficiently for either DNA or siRNA transfection. Furthermore the accumulation of positive charges at the cost of hydrophobic residues, for instance in dendrimers containing KK dyads in the G3 branch (1), G2 and G3 (2) or throughout the sequence (3) results in poor transfection efficiency.

The narrow range of peptide sequences compatible with efficient transfection is further illustrated for siRNA transfection with peptide dendrimers 15-20. Modifying 5 or $\mathbf{8}$ by placing the hydrophobic dipeptide (LL) in G2 instead of G1 (15 and 17), which reduces overall charge and increases hydrophobicity, almost completely suppresses knockdown. Acetylation of the N-termini (16) or extending the G3 branches with a histidine residue (18), which have a similar effect on charge and hydrophobicity, also decreases the knockdown efficiency. Interestingly, using histidine as cationic residue in G2,3-HL (19) lowers the knockdown properties, whereas having histidine in all three generations as for G1,2,3-HL (20) gives an almost as good knockdown as for G2,3-KL (5). Histidine residues being less protonated than lysine residues at neutral $\mathrm{pH}$, the peptide dendrimer probably requires a higher number of histidines to form a strong enough complex with siRNA for successful delivery.

\section{Cell Penetration and Nucleic Acid Complexation}

Transfection efficiency as measured by luciferase expression in the case of DNA or by GADPH knockdown in the case of siRNA reflects the overall process of nucleic acid complexation, cell penetration, and intracellular release. Cell penetration by the different dendrimer nucleic acid complexes can be quantified separately by fluorescence activated cell sorting (FACS) using fluorescence labeled nucleic acids, either a Cy5-DNA or siRNA-Cy3. These experiments show that the strongest internalization of DNA as measured by fluorescence intensity is achieved in complexes with G3-KL (4), although this dendrimer is a poor transfection reagent, followed by $\mathrm{G} 1,2,3-\mathrm{KL}$ (6), which in this case indeed is the best transfection reagent (Fig. 3A). In the case 
Table 1. Peptide dendrimers investigated for DNA and siRNA transfection

\begin{tabular}{|c|c|c|c|c|c|}
\hline No. & Short name & Sequence $^{\mathrm{a}}$ & Yield, mg $(\%)^{\mathrm{b}}$ & MS calc./obs. ${ }^{\mathrm{c}}$ & Ref. \\
\hline 1 & G3-KK & $(\mathrm{KK})_{8}(K \mathrm{LL})_{4}(K \mathrm{LL})_{2} K \mathrm{GSC}$ & $18.3(4)$ & $4570.2 / 4570.0$ & {$[23],[24]$} \\
\hline 2 & $\mathrm{G} 2,3-\mathrm{KK}$ & $(\mathrm{KK})_{8}(K \mathrm{KK})_{4}(K \mathrm{LL})_{2} K \mathrm{GSC}$ & $34.4(7)$ & $4690.3 / 4689.0$ & {$[23],[24]$} \\
\hline 3 & $\mathrm{G} 1,2,3-\mathrm{KK}$ & $(\mathrm{KK})_{8}(K \mathrm{KK})_{4}(K \mathrm{KK})_{2} K \mathrm{GSC}$ & $73.6(12)$ & $4750.3 / 4750.0$ & {$[23],[24]$} \\
\hline 4 & G3-KL & $(\mathrm{KL})_{8}(K \mathrm{LL})_{4}(K \mathrm{LL})_{2} K \mathrm{GSC}$ & $14.7(4)$ & $4450.0 / 4450.0$ & {$[23],[24]$} \\
\hline 5 & $\mathrm{G} 2,3-\mathrm{KL}$ & $(\mathrm{KL})_{8}(K \mathrm{KL})_{4}(K \mathrm{LL})_{2} K \mathrm{GSC}$ & $64.5(9)$ & $4510.1 / 4509.0$ & {$[23],[24]$} \\
\hline 6 & $\mathrm{G} 1,2,3-\mathrm{KL}$ & $(\mathrm{KL})_{8}(K \mathrm{KL})_{4}(K \mathrm{KL})_{2} K \mathrm{GSC}$ & $72.3(9)$ & $4540.1 / 4539.0$ & {$[23],[24]$} \\
\hline 7 & G3-RL & $(\mathrm{RL})_{8}(K \mathrm{LL})_{4}(K \mathrm{LL})_{2} K \mathrm{GSC}$ & $13.1(2)$ & $4674.2 / 4673.0$ & {$[23],[24]$} \\
\hline 8 & G2,3-RL & $(\mathrm{RL})_{8}(K \mathrm{RL})_{4}(K \mathrm{LL})_{2} K \mathrm{GSC}$ & $46.1(7)$ & $4846.3 / 4845.0$ & {$[23],[24]$} \\
\hline 9 & G1,2,3-RL & $(\mathrm{RL})_{8}(K \mathrm{RL})_{4}(K \mathrm{RL})_{2} K \mathrm{GSC}$ & $14.6(2)$ & $4932.3 / 4931.4$ & {$[23],[24]$} \\
\hline 10 & G1,2,3-KA & $(\mathrm{KA})_{8}(K \mathrm{KA})_{4}(K \mathrm{KA})_{2} K \mathrm{GSC}$ & $20.0(5)$ & $3951.0 / 3951.0$ & {$[23],[24]$} \\
\hline 11 & $\mathrm{G} 1,2,3-\mathrm{KH}$ & $(\mathrm{KH})_{8}(K \mathrm{KH})_{4}(K \mathrm{KH})_{2} K \mathrm{GSC}$ & $15.4(3)$ & $4875.9 / 4875.0$ & {$[23],[24]$} \\
\hline 12 & {$[\mathrm{G} 3-\mathrm{KL}]_{2}$} & {$\left[(\mathrm{KL})_{8}(K \mathrm{LL})_{4}(K \mathrm{LL})_{2} K \mathrm{GSC}\right]_{2}$} & $2.0(26)$ & $8898.1 / 8896.0$ & {$[23],[24]$} \\
\hline 13 & {$[\mathrm{G} 2,3-\mathrm{KL}]_{2}$} & {$\left[(\mathrm{KL})_{8}(K \mathrm{KL})_{4}(K \mathrm{LL})_{2} K \mathrm{GSC}\right]_{2}$} & $5.5(68)$ & $9018.2 / 9017.0$ & {$[23],[24]$} \\
\hline 14 & {$[\mathrm{G} 1,2,3-\mathrm{KL}]_{2}$} & {$\left[(\mathrm{KL})_{8}(K \mathrm{KL})_{4}(K \mathrm{KL})_{2} K \mathrm{GSC}\right]_{2}$} & $5.6(65)$ & $9078.2 / 9077.0$ & {$[23],[24]$} \\
\hline 15 & G1,3-KL & $(\mathrm{KL})_{8}(K \mathrm{LL})_{4}(K \mathrm{KL})_{2} K \mathrm{GSC}$ & $31.9(7)$ & $4480.1 / 4479.2$ & This work \\
\hline 16 & Ac-G2,3-KL & $(\mathrm{AcKL})_{8}(K \mathrm{KL})_{4}(K \mathrm{LL})_{2} K \mathrm{GSC}$ & $35.1(8)$ & $4846.4 / 4845.3$ & This work \\
\hline 17 & G1,3-RL & $(\mathrm{RL})_{8}(K \mathrm{LL})_{4}(K \mathrm{RL})_{2} K \mathrm{GSC}$ & $52.5(11)$ & $4760.1 / 4759.3$ & This work \\
\hline 18 & $\mathrm{H}-\mathrm{G} 2,3-\mathrm{KL}$ & $(\mathrm{HKL})_{8}(K \mathrm{KL})_{4}(K \mathrm{LL})_{2} K \mathrm{GSC}$ & $6.8(1)$ & $5607.2 / 5606.7$ & This work \\
\hline 19 & G2,3-HL & $(\mathrm{HL})_{8}(K \mathrm{HL})_{4}(K \mathrm{LL})_{2} K G S C$ & $5.6(1)$ & $4617.7 / 4616.8$ & This work \\
\hline 20 & G1,2,3-HL & $(\mathrm{HL})_{8}(K \mathrm{HL})_{4}(K \mathrm{HL})_{2} K \mathrm{GSC}$ & $19.0(4)$ & $4665.7 / 4664.8$ & This work \\
\hline
\end{tabular}

a One-letter code amino acids are used, $\mathrm{K}$ is the branching lysine residue, the $\mathrm{C}$-terminus at right is carboxamide, all $\mathrm{N}$-termini are free unless acetylated (16). ${ }^{b}$ All dendrimers are prepared by Fmoc strategy solid-phase peptide synthesis and purified by preparative reverse-phase HPLC. The yields given are isolated yield after collection of the purest fractions. 'Electrospray MS spectra were recorded on a Thermo Scientific LTQ Orbitrap XL.

of siRNA, the best transfection reagent G2,3-KL (5) shows good cell penetration, however comparable levels are also observed with other dendrimers that are less efficient for transfection, such as G3-KL (4) and G3-RL (7). Note that cell penetration is least efficient with G1,2,3-KL (6) and G1,2,3-RL (9), which are indeed not reactive as siRNA transfection reagents and only work for DNA (Fig. 3B).

The fact that certain dendrimers show efficient cell penetration as measured by FACS but lack good overall transfection efficiency can be explained by the strength of nucleic acid complexation as measured by heparin competition. Heparin is a polyanionic macromolecule which can compete with DNA or siRNA for binding to the dendrimers. The free nucleic acids are then detected by the PicoGreen fluorophore, which intercalates between base pairs and proportionally increases its fluorescence. This system allows us to see a difference in release between the different complexes and intrinsically the peptide dendrimers, which are the only changing component. For instance both G3-KL (4) and G3-RL (7) show a very strong DNA and siRNA complexation that cannot be displaced by heparin at any concentration, explaining their very good cell penetration but poor overall transfection efficiency (Fig. 4). By contrast G1,2,3-KL (6) and G1,2,3-RL (9) readily liberate their DNA when challenged with heparin, in line with the fact that these dendrimers are the best DNA transfection reagents in the series. Interestingly in the case of siRNA the best transfection reagents G2,3-KL (5) and G2,3-RL (8) form rather tight complexes with siRNA, which are only partially liberated upon competition with heparin (Fig. 4B).

It should be noted that the complexation of both DNA and siRNA using our dendrimer/lipofectin system results in slightly more compact nanoparticles compared to lipofectin alone. The effect is rather modest with DNA (contraction by
10-20\% compared to lipofectin alone) but is quite pronounced in the case of siRNA (contraction from $100 \mathrm{~nm}$ with lipofectin alone to approximately $50 \mathrm{~nm}$ when dendrimers G2,3-KL (5) or G1,2,3-KL (6) are present) (Fig. 5).

\section{Conclusion and Outlook}

Our initial studies with peptide dendrimers as transfection reagents as summarized above have rapidly established that efficient DNA and siRNA transfection is possible with peptide dendrimers when used in co-application with lipofectin. Well-defined structure-activity relationships were observed, with efficient transfection depending on the proper balance and placement of cationic and hydrophobic residues within the peptide dendrimer structure. Strikingly, optimizing transfection reactivity for DNA versus siRNA resulted in different peptide dendrimer 


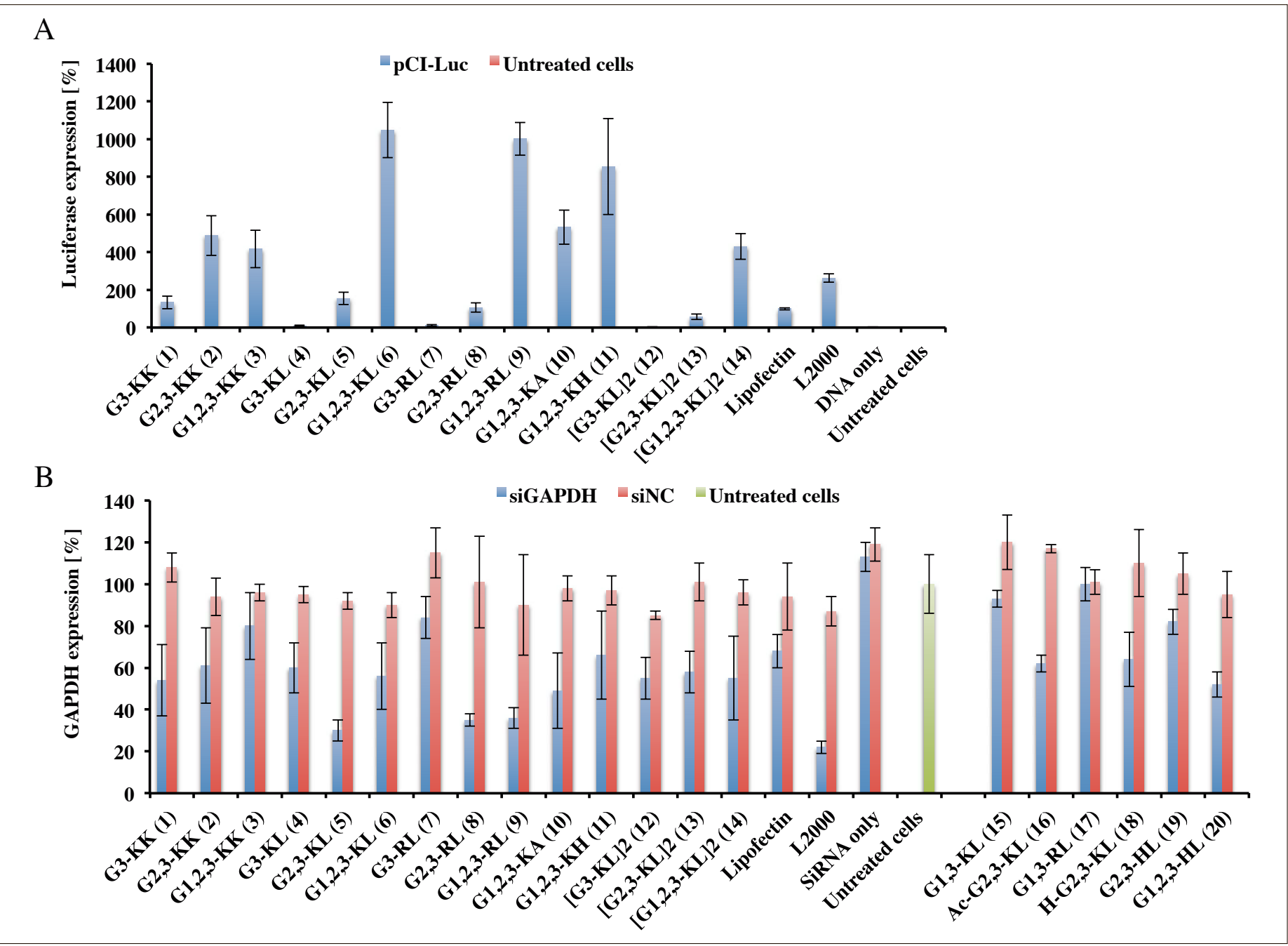

Fig. 2. Transfection efficiency of associated peptide dendrimer-lipofectin-DNA (A) or siRNA (B). Luciferase expression was quantified $24 \mathrm{~h}$ posttransfection and normalized to cells treated with a lipofectin-DNA complex (w/w 1:1) (A). GAPDH expression was quantified $48 \mathrm{~h}$ post transfection and normalized to untreated cells (B). Values are taken from refs $[23,24](\mathbf{1 - 1 4})$ and from this work (15-20). Error bars refer to the mean \pm SD for experiments carried out in triplicate of three independent experiments.

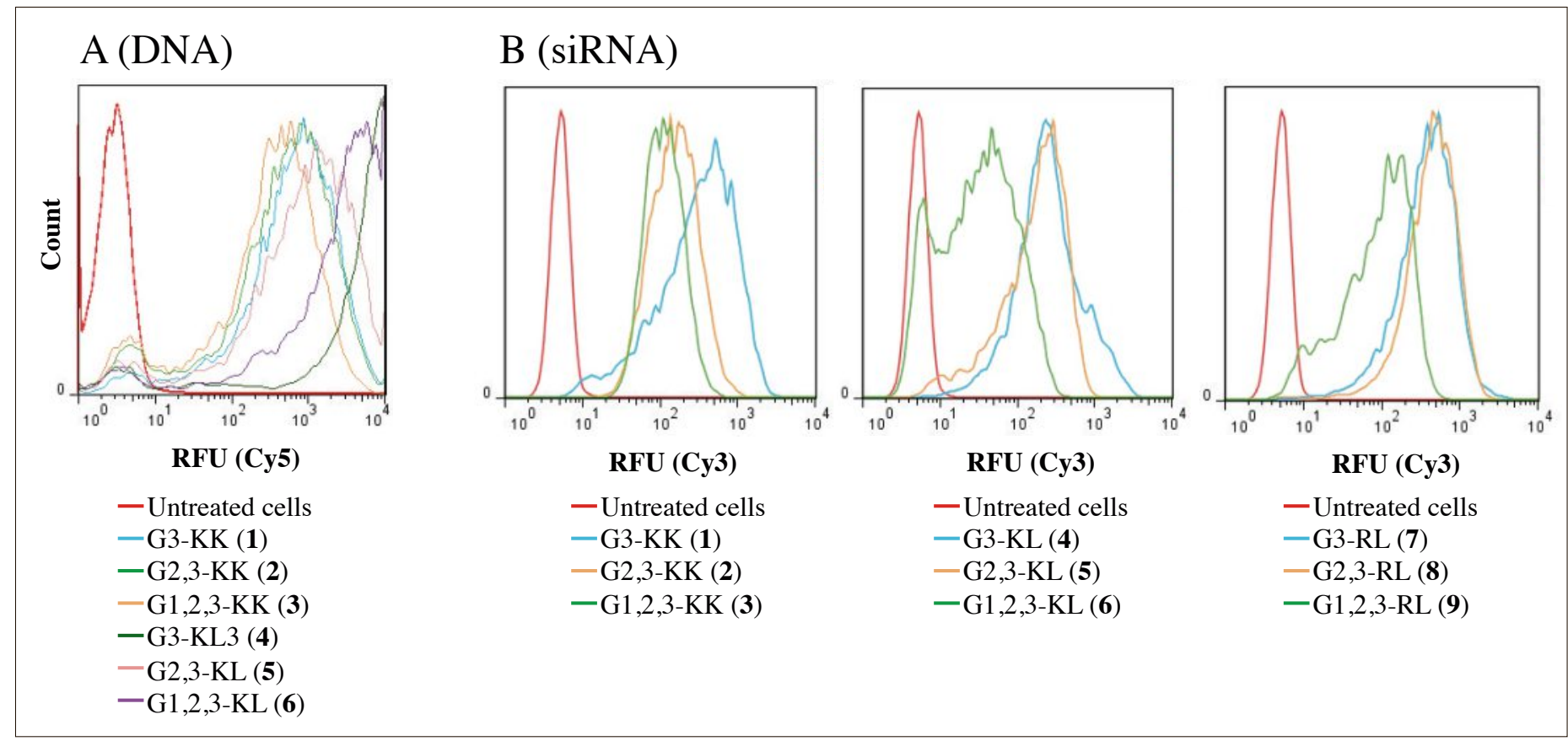

Fig. 3. Internalization efficiency of lipofectin-peptide dendrimer-DNA or siRNA complexes in HeLa cells. Complexes were formed with peptide dendrimer, lipofectin and labelled Cy5-DNA (A) or Cy3-siRNA (B) and transfected into HeLa cells as previously described. (RFU: relative fluorescence units). Figures taken from previously published studies. ${ }^{[23,24]}$ 
A

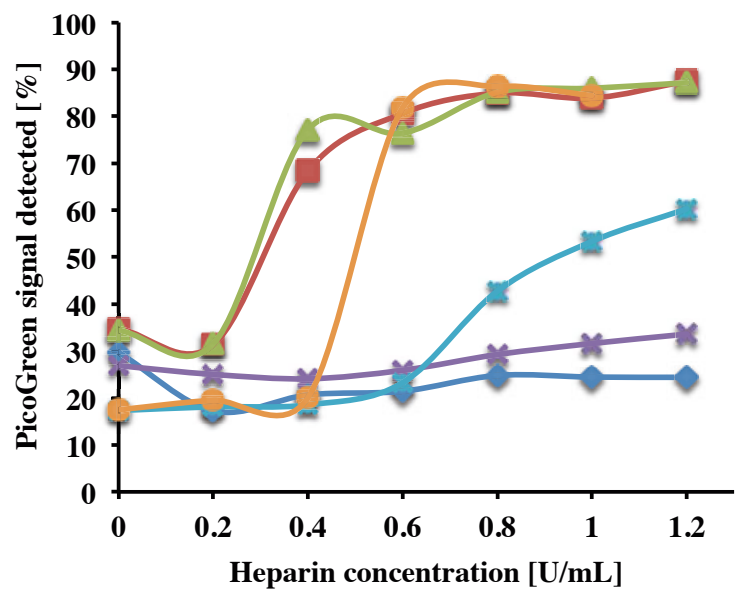

B

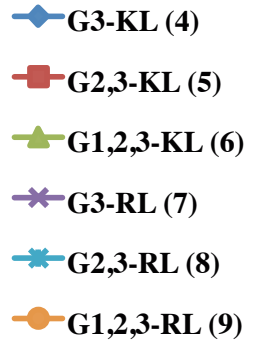

Fig. 4. Heparin competition assay of peptide dendrimer-lipofectin-DNA (A) or siRNA (B) complexes. Complexes were formed as mentioned above, then challenged to an increasing concentration of heparin in presence of PicoGreen. Data taken from refs [23,24].

sequences highlighting specific peptidenucleic acid interactions. The overall efficiency of our peptide dendrimer transfection reagents depends on efficient nucleic acid complexation, cellular penetration, and ultimately intracellular release of the target sequence. In contrast to most other transfection reagents, our peptide dendrimers have well-defined structures and can be synthesized in pure form and large quantities using the standard manufacturing processes available for peptides. Further optimization of these peptide dendrimers is currently ongoing to obtain systems with even higher transfection efficiencies and simpler chemical structures.

\section{Acknowledgements}

Marc Heitz is grateful to the Swiss Chemical Society and DSM for the SCS best poster presentation in chemical biology award. This work was supported by the University of Bern, the Swiss National Science Foundation (SNF), the Biotechnology and Biological Science Research Council (BBSRC), the Newton Trust, the European Research Council (ERC) and the EU Marie Curie ITN MMBio.

Received: January 26, 2017

1] F. H. C. Crick, Nature 1970, 227, 561.

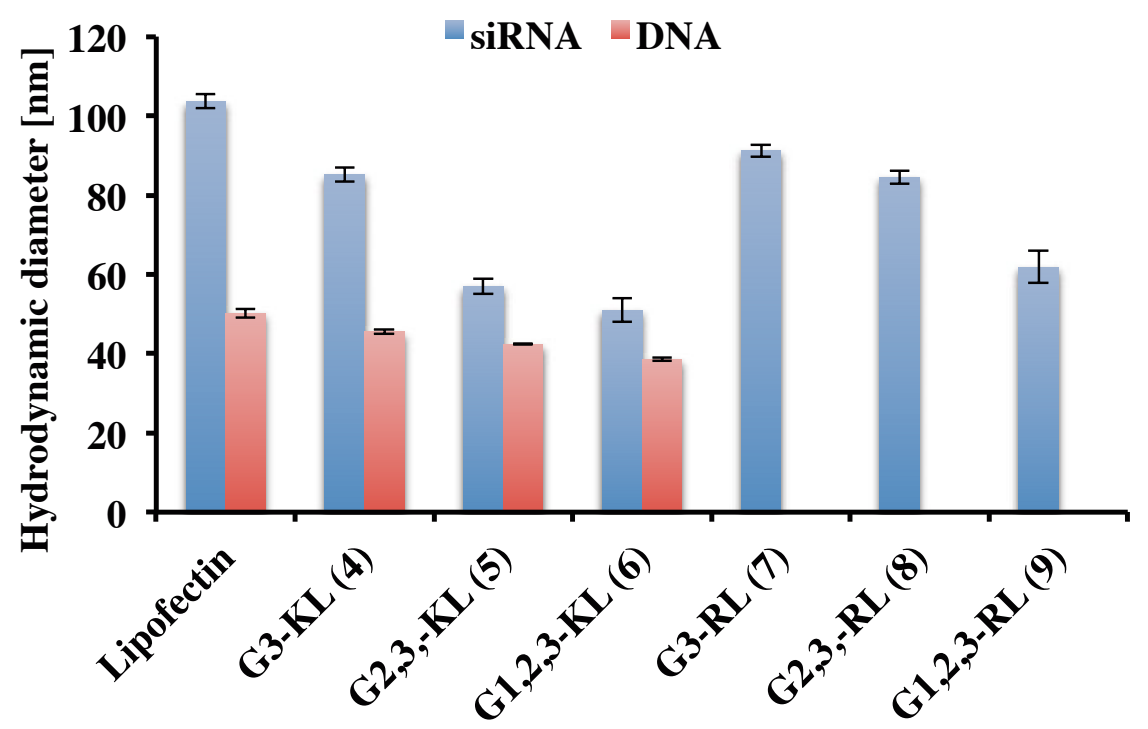

Fig. 5. Hydrodynamic diameters of the DNA or siRNA in complex with lipofectin and peptide dendrimers in $20 \mathrm{mM}$ HEPES buffer. Following $30 \mathrm{~min}$ incubation, the hydrodynamic diameter was recorded and analysed by dynamic light scattering with a Malvern Nano ZS. For each formulation, $\mathrm{n}=3-7$ and SDs are shown. All data shown have a polydispersity index $(\mathrm{PDI})<0.5$. Data taken from refs [23,24].

[2] A. Fire, S. Xu, M. K. Montgomery, S. A. Kostas, S. E. Driver, C. C. Mello, Nature 1998, $391,806$.

[3] S. M. Elbashir, J. Harborth, W. Lendeckel, A. Yalcin, K. Weber, T. Tuschl, Nature 2001, 411, 494.

[4] S. Sun, M. Wang, S. A. Knupp, Y. SotoFeliciano, X. Hu, D. L. Kaplan, R. Langer, D. G. Anderson, Q. Xu, Bioconjug. Chem. 2012, $23,135$.

[5] X. Guo, L. Huang, Acc. Chem. Res. 2012, 45, 971.

[6] S. C. Semple, A. Akinc, J. Chen, A. P. Sandhu, B. L. Mui, C. K. Cho, D. W. Y. Sah, D. Stebbing, E. J. Crosley, E. Yaworski, I. M. Hafez, J. R. Dorkin, J. Qin, K. Lam, K. G. Rajeev, K. F. Wong, L. B. Jeffs, L. Nechev, M. L. Eisenhardt, M. Jayaraman, M. Kazem, M. A. Maier, M. Srinivasulu, M. J. Weinstein, Q. Chen, R. Alvarez, S. A. Barros, S. De, S. K. Klimuk, T. Borland, V. Kosovrasti, W. L. Cantley, Y. K. Tam, M. Manoharan, M. A. Ciufolini, M. A. Tracy, A. de Fougerolles, I. MacLachlan, P. R. Cullis, T. D. Madden, M. J. Hope, Nat. Biotechnol. 2010, 28, 172.

[7] D. J. Gary, N. Puri, Y. Y. Won, J. Control. Release 2007, 121, 64. 
[8] S. An, D. He, E. Wagner, C. Jiang, Small 2015, 11,5142 .

[9] A. M. Chen, O. Taratula, D. Wei, H. Yen, T. Thomas, T. J. Thomas, T. Minko, H. He, ACS Nano 2010, 4, 3679.

[10] M. A. Mintzer, M. W. Grinstaff, Chem. Soc. Rev. 2011, 40, 173

[11] X. Liu, J. Zhou, T. Yu, C. Chen, Q. Cheng, K. Sengupta, Y. Huang, H. Li, C. Liu, Y. Wang, P. Posocco, M. Wang, Q. Cui, S. Giorgio, M. Fermeglia, F. Qu, S. Pricl, Y. Shi, Z. Liang, P. Rocchi, J. J. Rossi, L. Peng, Angew. Chem. Int. Ed. 2014, 53, 11822 .

[12] M. Wang, H. Liu, L. Li, Y. Cheng, Nat. Commun. 2014, 5, 3053

[13] K. Konate, M. F. Lindberg, A. Vaissiere, C. Jourdan, G. Aldrian, E. Margeat, S. Deshayes, P. Boisguerin, Int. J. Pharm. 2016, 509, 71 .

[14] Q. Tang, B. Cao, H. Wu, G. Cheng, PLoS One 2013, 8, e 54460 .
[15] Z. Tai, X. Wang, J. Tian, Y. Gao, L. Zhang, C. Yao, X. Wu, W. Zhang, Q. Zhu, S. Gao, Biomacromolecules 2015, 16, 1119.

[16] T. Asai, T. Tsuzuku, S. Takahashi, A. Okamoto, T. Dewa, M. Nango, K. Hyodo, H. Ishihara, H. Kikuchi, N. Oku, Biochem. Biophys. Res. Commun. 2014, 444, 599.

[17] C. C. Lee, J. a MacKay, J. M. J. Fréchet, F. C. Szoka, Nat. Biotechnol. 2005, 23, 1517.

[18] J. Kofoed, J. L. Reymond, Curr. Opin. Chem. Biol. 2005, 9, 656.

[19] T. Darbre, J. L. Reymond, Acc. Chem. Res. 2006, 39, 925.

[20] G. A. Eggimann, E. Blattes, S. Buschor, R. Biswas, S. M. Kammer, T. Darbre, J.-L. Reymond, Chem. Commun. (Camb). 2014, 50, 7254.

[21] J.-L. Reymond, T. Darbre, Org. Biomol. Chem. 2012, 10, 1483 .
[22] J.-L. Reymond, M. Bergmann, T. Darbre, Chem. Soc. Rev. 2013, 42, 4814.

[23] A. Kwok, G. A. Eggimann, J.-L. Reymond, T. Darbre, F. Hollfelder, ACS Nano 2013, 7, 4668.

[24] A. Kwok, G. A. Eggimann, M. Heitz, J.L. Reymond, F. Hollfelder, T. Darbre, ChemBioChem 2016, 17, 2223.

[25] N. Maillard, A. Clouet, T. Darbre, J.-L. Reymond, Nat. Protoc. 2009, 4, 132.

[26] J. H. Felgner, R. Kumar, C. N. Sridhar, C. J. Wheeler, Y. J. Tsai, R. Border, P. Ramsey, M. Martin, P. L. Felgner, J. Biol. Chem. 1994, 269 , 2550.

[27] S. J. Gould, S. Subramani, Anal. Biochem. 1988, 175,5

[28] A. Kwok, S. L. Hart, Nanomed. Nanotechnol. Biol. Med. 2011, 7, 210.

[29] R. D. Barber, D. W. Harmer, R. A. Coleman, B. J. Clark, Physiol. Genomics 2005, 21, 389. 